\title{
Mm-wave $\mathrm{HCO}^{+}, \mathrm{HCN}$ and CO absorption toward NGC 1052
}

\author{
H. Liszt ${ }^{1}$ and R. Lucas ${ }^{2}$ \\ 1 National Radio Astronomy Observatory, 520 Edgemont Road, Charlottesville, VA, 22903-2475, USA \\ e-mail: hliszt@nrao.edu \\ ${ }^{2}$ Institut de Radioastronomie Millimétrique, 300 rue de la Piscine, 38406 Saint Martin d'Hères, France
}

Received 12 July 2004 / Accepted 26 August 2004

\begin{abstract}
We used the Plateau de Bure Interferometer to observe $\lambda 3 \mathrm{~mm} J=1-0$ absorption lines of $\mathrm{HCO}^{+}, \mathrm{HCN}$ and $\mathrm{CO}$ toward the core of the nearby elliptical, megamaser-host galaxy NGC 1052. The lines are relatively weak, with peak optical depths 0.03 for $\mathrm{HCO}^{+}$and $\mathrm{HCN}$ and 0.1 for $\mathrm{CO}$. Nonetheless the inferred column density of molecular gas $2 N\left(\mathrm{H}_{2}\right) \simeq 5 \times$ $10^{21} \mathrm{~cm}^{-2}$ is consistent with the degree of reddening inferred toward the nucleus from observations of the Balmer series of hydrogen. Mm-wave absorption line profiles are somewhat broader than those of $\mathrm{H} \mathrm{I}$ and $\mathrm{OH}$, perhaps because lower free-free opacity at mm-wavelengths exposes higher-velocity material nearer the nucleus. Overall, the $\mathrm{OH} / \mathrm{HCO}^{+}$ratio in $\mathrm{NGC} 1052$ is as expected from the strong relationship established in local diffuse clouds but the optical depth ratio varies strongly over the line profiles. Similar variations are also seen toward Cen A, which has very different line ratios among $\mathrm{H} \mathrm{I}, \mathrm{OH}$ and $\mathrm{HCO}^{+}$for very nearly the same amount of $\mathrm{OH}$ absorption.
\end{abstract}

Key words. galaxies: ISM - ISM: molecules - galaxies: elliptical and lenticular, cD

\section{Introduction}

NGC 1052 is a nearby elliptical galaxy $\left(v_{\mathrm{sys}}=1480 \mathrm{~km} \mathrm{~s}^{-1}\right.$; $1^{\prime \prime}=102.5 \mathrm{pc}$ for $H_{0}=70 \mathrm{~km} \mathrm{~s}^{-1} \mathrm{Mpc}^{-1}$ ) with a variety of distinguishing nuclear characteristics. It hosts a weak LINER active galactic nucleus (AGN) (Gabel et al. 2000) with a pc-scale VLBI jet-counterjet structure visible up to $43 \mathrm{GHz}$ (Vermeulen et al. 2003). An $\mathrm{H}_{2} \mathrm{O}$ megamaser is seen against the counter-jet (Claussen et al. 1998) and NGC 1052 is the only case in which an elliptical galaxy is known to host such phenomena: the other elliptical cited by Claussen et al. (1998), TXSS 2226-184 (Koekemoer et al. 1995), has recently been shown likely to be a disk+bulge system (Falcke et al. 2000). On kpc-scales one finds a dust lane and a pair of complementary counter-rotating nuclear gas disks (Plana \& Boulesteix 1996) as well as a double radio source (Wrobel 1984). An extended H I envelope about NGC 1052 was found by van Gorkom et al. (1986) and Omar et al. (2002) recently discovered $\mathrm{OH}$ absorption against the nucleus as well. $\mathrm{OH}$ absorption over a wider range of velocity was found by Vermeulen et al. (2003), who also present a comprehensive study of H I absorption.

Weak absorption against the nuclear continuum complicates interpretation of the $\mathrm{H}$ I emission over the optical disk of the galaxy but the column densities seen in $21 \mathrm{~cm}$ emission at relatively low resolution $\left(1^{\prime} \approx 6 \mathrm{kpc}\right)$ are moderate, of or$\operatorname{der} N(\mathrm{H}) \simeq 10^{20} \mathrm{~cm}^{-2}$ (Fig. 5 of van Gorkom et al. 1986). The hydrogen column densities inferred from the presence of $\mathrm{OH}$ are larger than those derived from the $\mathrm{H}$ I emission, consistent with obvious optical obscuration and the inference of nuclear reddening (albeit with substantial uncertainty) $E_{B-V}=0.42 \pm$ 0.2 mag. by Gabel et al. (2000), implying $N(\mathrm{H}) \simeq 2.5 \times$ $10^{21} \mathrm{~cm}^{-2}$ for conditions like those seen locally in the disk of the Milky Way.

In the Milky Way, the detection of $\mathrm{cm}$-wave $\mathrm{OH}$ absorption is a sure indicator of the presence of other trace molecules, especially $\mathrm{HCO}^{+}$(Lucas \& Liszt 1996; Liszt \& Lucas 1996), CO (Liszt \& Lucas 1998), $\mathrm{C}_{2} \mathrm{H}$ (Lucas \& Liszt 2000a), and (somewhat more occasionally) CN and HCN (Liszt \& Lucas 2001), even in diffuse clouds. Given this, we sought and found mmwave absorption from $\mathrm{HCO}^{+}, \mathrm{HCN}$ and $\mathrm{CO}$ toward the nucleus of NGC 1052. The implications of these observations, and a comparison with Cen A (NGC 5128; the only other elliptical where the same absorption lines have been observed) are the subject of this work. Section 2 describes the observations and a discussion is given in Sect. 3 .

\section{Mm-wave observations of $\mathrm{HCO}^{+}, \mathrm{HCN}$ and $\mathrm{CO}$}

The $J=1-0$ transitions of $\mathrm{HCN}(88.631 \mathrm{GHz}), \mathrm{HCO}^{+}$ $(89.188 \mathrm{GHz})$ and $\mathrm{CO}(115.271 \mathrm{GHz})$ were observed at the Plateau de Bure Interferometer at various times during the year 2001. The new spectra discussed here, shown in Fig. 1, were taken with $2.5 \mathrm{MHz}$ wide correlator channels corresponding to $8.46,8.50$ and $6.5 \mathrm{~km} \mathrm{~s}^{-1}$ for $\mathrm{HCN}, \mathrm{HCO}^{+}$and $\mathrm{CO}$ respectively. The passbands were much wider than the excerpts shown here. Reduction of such data, where a strong unresolved background source is also the calibrator, is basically a matter of accumulating line/continuum ratios. The continuum 


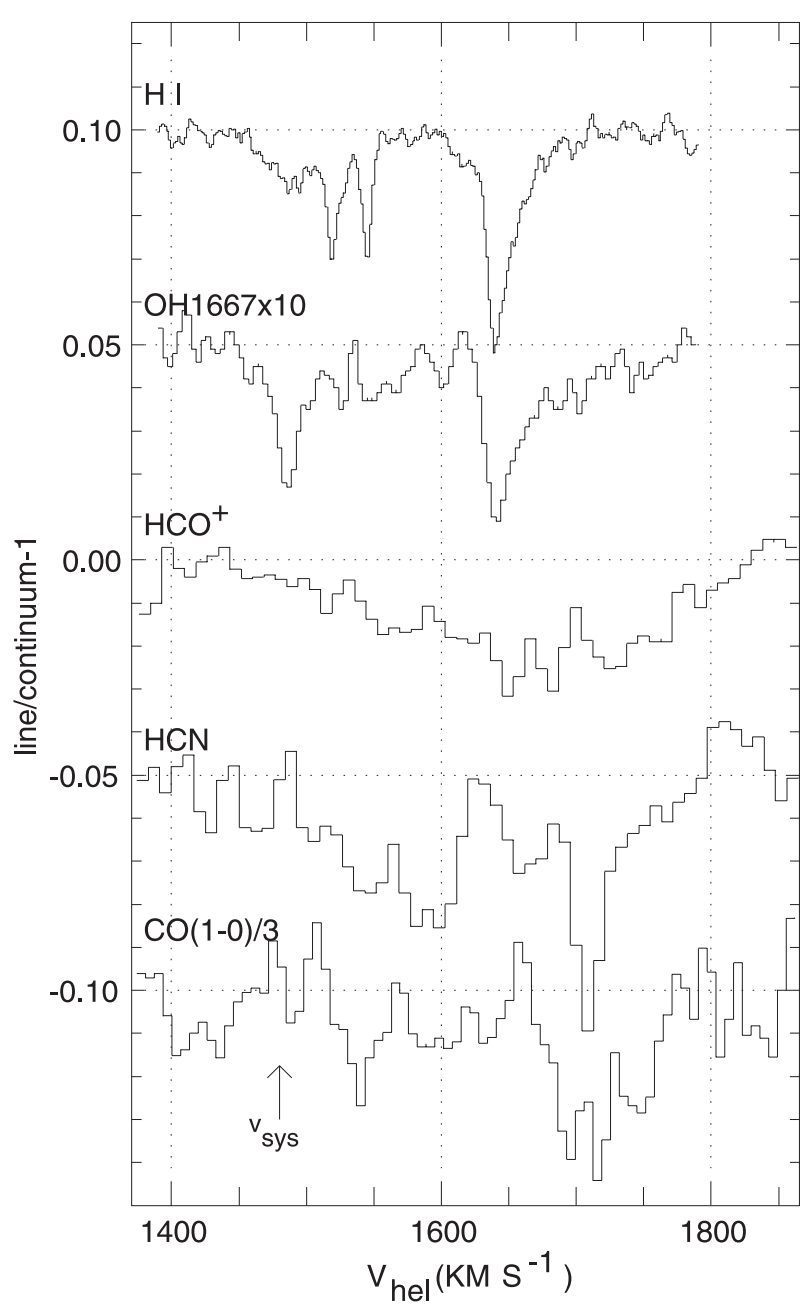

Fig. 1. Absorption profiles toward NGC 1052. The data are presented as scaled, offset, line/continuum ratios. The $\mathrm{H} \mathrm{I}$ and $\mathrm{OH}$ profiles at top are the "total" VLB profiles from Vermeulen et al. (2003) representing spatially-resolved data averaged over some 15-20 mas or $1.5-2.0 \mathrm{pc}$ at one epoch. Profiles of the $J=1-0$ transitions of $\mathrm{HCN}$, $\mathrm{HCO}^{+}$and $\mathrm{CO}$ from the Plateau de Bure represent data taken at multiple epochs against the vastly-unresolved mm-wave continuum, whose spatial distribution is compact and probably similar to that at lower frequencies, but with a larger contribution from regions at smaller radii (see Sect. 3.1 of the text).

level, approximately 0.6 Jy, was not precisely determined, however. Spectral baselines in the data were determined from the feature-free regions alongside the spectra.

The column density in the lowest $(J=0)$ level of a simple linear molecule is related to the integrated optical depth of the $J=1-0$ transition as

$N_{0}=\frac{8.0 \times 10^{12} \mathrm{~cm}^{-2} \int \tau_{10} \mathrm{~d} v}{\mu^{2}\left(1-\exp \left(-h v_{10} / k T_{\text {exc }}\right)\right)}$

where $\mu$ is the permanent dipole moment of the molecule, $T_{\operatorname{exc}}$ is the excitation temperature of the $J=1-0$ transition, $v_{10}$ is its frequency and the profile integral is in units of $\mathrm{km} \mathrm{s}^{-1}$. The dipole moments of the species observed here are $2.98(\mathrm{HCN})$, $4.07\left(\mathrm{HCO}^{+}\right)$and 0.112 Debye. Except in fairly dense gas, the excitation of $\mathrm{HCN}$ and $\mathrm{HCO}^{+}$is seen to be very weak (Lucas \& Liszt 1996; Liszt \& Lucas 1996) and the column density can be reliably determined from observation of the $J=1-0$ line. Denoting the $J=1-0$ optical depth profile integral as $I_{10}$ (units of $\mathrm{km} \mathrm{s}^{-1}$ ) and taking the excitation temperature as $2.73 \mathrm{~K}$, one finds $N(\mathrm{HCN})=1.92 \times 10^{12} \mathrm{~cm}^{-2} I_{10}, N\left(\mathrm{HCO}^{+}\right)=1.02 \times$ $10^{12} \mathrm{~cm}^{-2} I_{10}$ and $N(\mathrm{CO})=1.03 \times 10^{15} \mathrm{~cm}^{-2} I_{10}$. For an excitation temperature of $10 \mathrm{~K}, N(\mathrm{HCN})=1.31 \times 10^{13} \mathrm{~cm}^{-2} I_{10}$, $N\left(\mathrm{HCO}^{+}\right)=6.96 \times 10^{12} \mathrm{~cm}^{-2} I_{10}$ and $N(\mathrm{CO})=5.96 \times$ $10^{15} \mathrm{~cm}^{-2} I_{10}$.

Below we also consider the column density of $\mathrm{OH}$ determined from observation of the $1667 \mathrm{MHz}$ line, which for LTE conditions is written $N(\mathrm{OH})=2.24 \times$ $10^{14} \mathrm{~cm}^{-2} T_{\text {exc }} \int \tau \mathrm{d} v$. For diffuse clouds locally this is believed to be a good approximation, and the excitation is found to be quite weak, $T_{\text {exc }}-T_{\mathrm{cmb}} \simeq 1 \mathrm{~K}$ (Liszt \& Lucas 1996; Felenbok \& Roueff 1996).

\subsection{Dense or diffuse/translucent gas?}

The present work discusses the observations in terms of diffuse/translucent gas of low true optical depth and a fullycovered continuum, which seems reasonable given the small size of the latter - at most $2 \mathrm{pc}$ and perhaps much less if the core is exposed at/above $90 \mathrm{GHz}$; see Sect. 3. This provides a consistent and rather compact interpretation with a minimum of argument and uncertainty. However, nothing in the presentlyavailable data absolutely refutes the alternative that the gas is dense and has higher optical depth and low covering factor, at least in part. For instance, the excitation temperature of the CO 1-0 transition overall could be $100 \mathrm{~K}$, rather than $6 \mathrm{~K}$, if the expected amount of gas phase carbon is to be put in dense, rather than diffuse, gas (see Sect. 3). Observations of higher-lying mm-wave transitions might help to settle this issue, though the rapid evolution of the continuum with epoch and observing frequency will complicate matters.

\subsection{Borrowed and previously-published spectra}

We are indebted to several authors for providing copies of their previously-published data. For reference, they are: toward NGC 1052, H I and $\mathrm{OH}$ absorption profiles from Vermeulen et al. (2003), and toward Cen A, H I from Sarma et al. (2002), $\mathrm{OH}$ from van Langevelde et al. (1995) and $\mathrm{HCO}^{+}$from Wiklind \& Combes (1997). A datacube of $\mathrm{OH}$ absorption profiles seen in the Milky Way near Sgr A* originally taken by Killeen et al. (1992) is referenced in Sect. 3. The CO $J=3-2$ emission profile toward Cen A in Fig. 2 is from Liszt (2001) and was taken at the $\mathrm{JCMT}$ with $\simeq 14^{\prime \prime}$ resolution.

\section{MM-wave absorption toward NGC 1052}

The new mm-wave absorption data are summarized in Table 1 and shown in Fig. 1. Several strong and distinct features seen in $\mathrm{OH}$ and $\mathrm{H} \mathrm{I}$ are not apparent at mm-wavelengths. The $\mathrm{CO}$ and $\mathrm{HCN}$ profiles seem to resemble each other more closely than the $\mathrm{HCO}^{+}$. The latter, which has by far the lowest noise in our dataset, is somewhat broader than the $\mathrm{OH}$, which is in turn noticeably more extensive than H I. As discussed in Sect. 3.2 


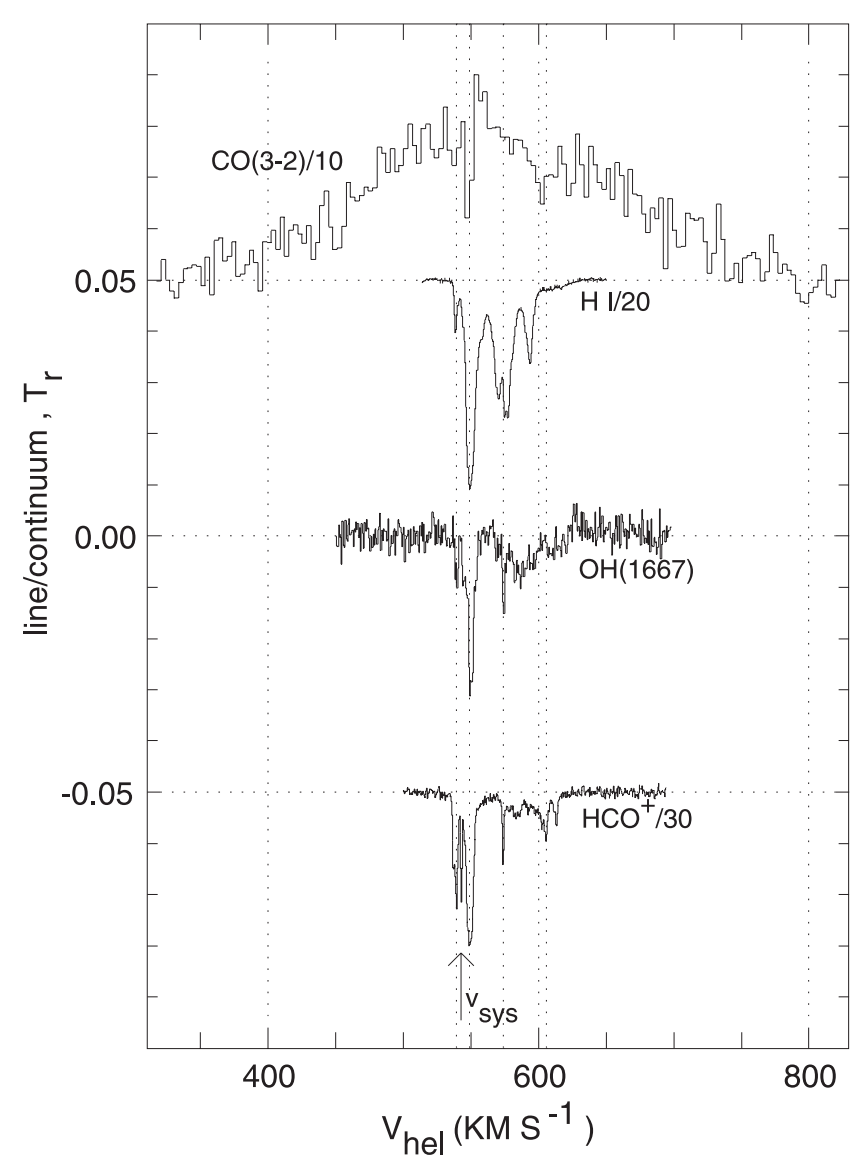

Fig. 2. CO $J=3-2$ emission and various absorption profiles toward Centaurus A. The CO is the centermost profile in the study of Liszt (2001). The H I is from Sarma et al. (2002), OH from van Langevelde et al. (1995) and $\mathrm{HCO}^{+}$from Wiklind \& Combes (1997).

Table 1. Integrated optical depth: NGC 1052.

\begin{tabular}{lcccc}
\hline \hline Line & $\sigma_{l / c}^{1}$ & Low $v^{2}$ & High $v$ & Total \\
\hline & & $\mathrm{km} \mathrm{s}^{-1}$ & $\mathrm{~km} \mathrm{~s}^{-1}$ & $\mathrm{~km} \mathrm{~s}^{-1}$ \\
\hline $\mathrm{H} \mathrm{I}^{3}$ & 0.0017 & $1.34(0.02)$ & $1.63(0.02)$ & $2.97(0.03)$ \\
$\mathrm{OH}^{3}$ & 0.0036 & $0.153(0.008)$ & $0.213(0.009)$ & $0.365(0.012)$ \\
$\mathrm{HCN}$ & 0.0086 & $2.89(0.40)$ & $3.25(0.40)$ & $6.15(0.58)$ \\
$\mathrm{HCO}^{+}$ & 0.0037 & $1.50(1.12)$ & $4.11(0.12)$ & $5.47(0.18)$ \\
$\mathrm{CO}$ & 0.027 & $3.64(1.13)$ & $9.92(1.15)$ & $13.56(1.61)$ \\
\hline
\end{tabular}

${ }^{1} \sigma_{l / c}^{1}$ is the rms noise in the line/continuum ratio.

${ }^{2} v<1600 \mathrm{~km} \mathrm{~s}^{-1}$.

${ }^{3}$ Data from Vermeulen et al. (2003).

(see Fig. 2), the various species seen toward Cen A exhibit quite similar behaviour.

The differences between $\mathrm{H} \mathrm{I}$ and $\mathrm{OH}$ which occur in the two ellipticals are quite typical of what is seen locally along rather random directions toward extragalactic point radio continuum sources (Liszt \& Lucas 1996), reflecting the localization and/or segregation of cool and diffuse molecular gas in the nearby ISM. As such, they do not necessarily signal differences between nuclear and off-nuclear or dense and diffuse gas toward NGC 1052. Similarly, sharp differences between the absorption profiles of $\mathrm{HCO}^{+}$and species like HCN (Liszt \& Lucas 2001) or CO (Liszt \& Lucas 1998) arise along local lines of sight because of differing stages of chemical maturity in the various diffuse clouds. However, differences between $\mathrm{HCO}^{+}$and $\mathrm{OH}$ absorption profiles generally do not occur in the local ISM and this issue is addressed separately in Sects. 3.1 and 3.3.

The $\mathrm{HCO}^{+}$profile integral converted to column density in the low-excitation limit yields $N\left(\mathrm{HCO}^{+}\right)=5.5 \times 10^{12} \mathrm{~cm}^{-2}$, implying $2 N\left(\mathrm{H}_{2}\right)=3.6-5.6 \times 10^{21} \mathrm{~cm}^{-2}$ for $\mathrm{HCO}^{+} / \mathrm{H}_{2}$ ratios $X\left(\mathrm{HCO}^{+}\right)=2-3 \times 10^{-9}$ typical of galactic diffuse and translucent gas. Locally, such a gas column would imply a reddening of $E_{B-V}=0.6-1.0$ mag which is consistent with the inference of Gabel et al. (2000) that $E_{B-V}=0.42 \pm 0.2$ mag. The same exercise may be repeated with $\mathrm{OH}$, which in diffuse and translucent gas has a relatively stable relative abundance $X(\mathrm{OH})=10^{-7}$ (Liszt \& Lucas 1996, 2002). For an excitation temperature of $4 \mathrm{~K}, N(\mathrm{OH})=3.27 \times 10^{14} \mathrm{~cm}^{-2}$ implying $2 N\left(\mathrm{H}_{2}\right)=6.5 \times 10^{21} \mathrm{~cm}^{-2}$. The consistency of these estimates implies that the mean $\mathrm{OH} / \mathrm{HCO}^{+}$opacity ratio is similar to that seen in the Milky Way as well (see Sect. 3.2).

The column density ratio $N(\mathrm{HCN}) / N\left(\mathrm{HCO}^{+}\right)=2.1$ is entirely typical of local gas (Liszt \& Lucas 2001); this ratio is relatively unaffected by assumptions about the overall excitation, although $\mathrm{HCO}^{+}$is somewhat more easily excited in diffuse gas. By contrast, carbon monoxide is unlikely to remain as cold rotationally as the $\mathrm{HCO}^{+}$. The $\mathrm{CO}$ column density is $1.4<N(\mathrm{CO})<8.1 \times 10^{16} \mathrm{~cm}^{-2}$ for $2.73 \mathrm{~K}<T_{\text {exc }}<10 \mathrm{~K}$. $\mathrm{CO} / \mathrm{HCO}^{+}$ratios as large as that implied by the upper limit $\mathrm{CO}$ column density are rather high for diffuse gas (Liszt \& Lucas 1998). Even so, the $\mathrm{CO} / \mathrm{H}$ ratio $N(\mathrm{CO}) / 2 N\left(\mathrm{H}_{2}\right) \lesssim 8.1 \times$ $10^{16} \mathrm{~cm}^{-2} / 5 \times 10^{21} \mathrm{~cm}^{-2} \simeq 10^{-5}$ is still about one order of magnitude too small to account for the bulk of the free gasphase carbon expected for material having Solar metallicity and carbon depletion onto grains like that seen locally, where $[\mathrm{C}] /[\mathrm{H}]=1.4 \times 10^{-4}$ (Savage \& Sembach 1996).

The absence of most of the expected carbon from $\mathrm{CO}$ suggests either low metallicity or diffuse molecular gas, see Liszt \& Lucas (1998) or Fig. 9 of Liszt \& Lucas (2000). However, assuming a gas of lower metallicity would probably still not account for all the gas phase carbon, because the $\mathrm{OH} / \mathrm{H}_{2}$ and $\mathrm{HCO}^{+} / \mathrm{H}_{2}$ ratios used to derive $N\left(\mathrm{H}_{2}\right)$ would presumably be smaller in a gas of lower metallicity, thereby increasing the inferred $N\left(\mathrm{H}_{2}\right)$. Nonetheless, there is a somewhat indirect suggestion of sub-solar metallicity, found in another context. Gabel et al. (2000) noted (see their Sect. 5) that some anomalies in their photoionization models would be alleviated if the $220 \mathrm{~nm}$ bump were not present in the $u v$ extinction curve. This is typical of SMC-like extinction curves (Pei et al. 1991).

The observed CO $J=1-0$ optical depth, if it arises in a gas with $T_{\text {exc }}=10 \mathrm{~K}$, would produce an emission line with a brightness temperature $0.5 \mathrm{~K}$ and an integrated profile brightness $40 \mathrm{~K} \mathrm{~km} \mathrm{~s}^{-1}$. Combined with a galactic $\mathrm{CO} / \mathrm{H}_{2}$ conversion factor, such an integrated brightness would imply $2 \mathrm{~N}\left(\mathrm{H}_{2}\right) \simeq$ $40 \times 4 \times 10^{20} \mathrm{~cm}^{-2}$. This is a factor 2-3 above the estimates of $2 \mathrm{~N}\left(\mathrm{H}_{2}\right)$ from $\mathrm{HCO}^{+}$and $\mathrm{OH}$. However, the brightness of any CO $J=1-0$ emission from NGC 1052 is not as large as $0.02 \mathrm{~K}$ (Wiklind et al. 1995) over a $25^{\prime \prime}$ beam.

From these arguments we draw several conclusions. The excitation temperature of the $\mathrm{CO}$ is probably smaller than $10 \mathrm{~K}$; 
taking $T_{\text {exc }}=6 \mathrm{~K}$, implying $N(\mathrm{CO})=3.5 \times 10^{16} \mathrm{~cm}^{-2}$ would lower the integrated brightness by a factor two with respect to the estimate in the preceding paragraph, reconciling the estimates of $N\left(\mathrm{H}_{2}\right)$ from the various trace molecules. Secondly, the $\mathrm{CO}$ gas producing the observed absorption cannot fill more than a small fraction of the $10^{\prime \prime}-60^{\prime \prime}$ singledish telescope beams which have been used to search for $\mathrm{CO}$ emission; crudely, at most of order $0.02 \mathrm{~K} / 0.25 \mathrm{~K}$. However, at this level, the line emission flux into a singledish beam may not be large compared to the continuum flux which is being absorbed out by the gas we have detected. That is, $10 \%$ absorption of an $0.6 \mathrm{Jy}$ continuum, observed by a telescope with $6 \mathrm{Jy} / \mathrm{K}$ gain, produces a negative signal of $0.1 \times 0.6 / 6=0.01 \mathrm{~K}$. Thus, searches for $\mathrm{CO}$ emission have probably been compromised somewhat by the same confusion between emission and absorption which afflicted the H I synthesis of van Gorkom et al. (1986). Off-nuclear searches for CO emission in the optical dust lane would avoid this problem.

The inferred molecular column densities are much higher than those of $\mathrm{H}$ I seen in emission about the optical disk of NGC 1052, $N(\mathrm{H} \mathrm{I}) \simeq 10^{20} \mathrm{~cm}^{-2}$ (van Gorkom et al. 1986). However, the $\mathrm{H}$ I absorption column density implied by the integrated optical depth, $N(\mathrm{H} \mathrm{I})=5.3 \times 10^{20} T_{\mathrm{sp}} / 100 \mathrm{~K}$, is also quite likely to be much larger than that seen in the lowresolution emission data. In the absence of much higher resolution H I emission data, very little can be said about the actual spin temperature in the atomic gas, except to note that the amount of $\mathrm{H} \mathrm{I}$ absorption which is seen is substantially higher than would be produced by extra-nuclear foreground gas having $N(\mathrm{H} \mathrm{I})=10^{20} \mathrm{~cm}^{-2}$ at a typical $T_{\mathrm{sp}}=100 \mathrm{~K}$, implying that the $\mathrm{H}$ I absorption does in fact arise nearer the nucleus. Conversely, the $\mathrm{H} \mathrm{I}$ absorption column density is still too small to account for the observed reddening unless the spin temperature is extraordinarily high. It seems more likely that the gas associated with the bulk of the occulting column density is that sampled by the trace molecules.

\subsection{Kinematics}

If the $\mathrm{HCO}^{+}$profile is wider and generally different in shape than the $\mathrm{OH}$, such differences are not expected to arise chemically - as when comparing $\mathrm{OH}$ or $\mathrm{HCO}^{+}$with $\mathrm{H} \mathrm{I}$ - but may occur for any of several other reasons. The optical depth of the $1667 \mathrm{MHz} \mathrm{OH}$ line is subject to excitation effects which produce rather spectactular results at higher $\mathrm{OH}$ column densities between $10^{14}-10^{15} \mathrm{~cm}^{-2}$ (van Langevelde et al. 1995) but $\mathrm{OH}$ exists in phases of lower and higher excitation even in diffuse gas (Liszt \& Lucas 1996). The $\mathrm{OH}-\mathrm{HCO}^{+}$comparison is of particular importance and is discussed separately in Sect. 3.3.

Another effect which may be important in NGC 1052 is the frequency dependence of the free-free opacity about the nucleus. Kameno et al. (2001) have modelled an asymmetric electron distribution having a peak free-free opacity of 300 toward the core at $15.4 \mathrm{GHz}$. Scaling as $v^{-2.1}$, the peak opacity at $89-115 \mathrm{GHz}$ would be only $4-7$. Thus the innermost parts of the jets could become visible in mm-wave absorption (there is a pronounced gap in VLB maps up to $43 \mathrm{GHz}$, see
Vermeulen et al. 2003), perhaps exposing higher-velocity foreground material to illumination by background continuum.

Another possibility for creating differences is the secular evolution of the blobby knot structure within the VLB jet; the few years which elapsed between the observations of Vermeulen et al. (2003) and our own (from 1997 and 1998 to 2001) most likely witnessed substantial evolution in the jet structure. Spectral index variations over the jet structure will also result in different weighting of absorption from foreground gas seen at widely differing frequencies.

The kinematics of the inner regions are rather poorly understood. Redshifted gas appears on both sides of the nucleus, to the West in the $\mathrm{H}_{2} \mathrm{O}$ maser spots seen against the counterjet and to the East and West in H I absorption (Claussen et al. 1998; Vermeulen et al. 2003). The $\mathrm{H} \alpha$ line (Plana \& Boulesteix 1996) shows complementary prograde and retrograde rotating disks on scales of 1-2 kpc $\left(10^{\prime \prime}-20^{\prime \prime}\right)$ but the velocities of neither structure (relative to the systemic) approach those seen in the masers or radiofrequency absorption lines. This is analogous to Cen A, where the nuclear disk seen in $\mathrm{CO}$ emission (Liszt 2001) has no presence whatsoever in $\mathrm{H} \alpha$ (Bland et al. 1987). The differences between $\mathrm{CO}$ and $\mathrm{H} \alpha$ in the nucleus of Cen A stand in sharp contrast to what is seen over the larger dust lane, where they behave essentially identically within the various limitations of the observational data. However, the circumnuclear disk in Cen A is well-described as purely rotating (Liszt 2001), which cannot account for the redshifted absorption features.

\subsection{Comparison with Cen $A$}

Shown in Fig. 2 are profiles toward Cen A like those shown in Fig. 1 for NGC 1052. As toward NGC 1052, the absorption is red-shifted with respect to the systemic velocity, the most extreme velocities (relative to the systemic velocity) are more prominent in molecular gas, and several very prominent, discrete $\mathrm{H}$ I features have no counter part in molecular absorption. The somewhat eccentric placement of the various narrow $\mathrm{OH}$ and $\mathrm{HCO}^{+}$components with respect to $\mathrm{H} \mathrm{I}$ are entirely typical of local lines of sight through the Milky Way (Lucas \& Liszt 1996; Liszt \& Lucas 1996).

Until recently it appeared that $\mathrm{H} \mathrm{I}$ absorption was considerably less extended in velocity, but the broad, faint wing above $600 \mathrm{~km} \mathrm{~s}^{-1}$ discovered by Sarma et al. (2002) corrects this misimpression. Despite its substantial width, all of the absorption toward Cen $\mathrm{A}$ is much narrower than even the redshifted portion of the $\mathrm{CO}$ emission profile, which represents contributions from both the dust lane and inner circumnuclear disk (Liszt 2001).

\section{3. $\mathrm{OH}$ and $\mathrm{HCO}^{+}$in $\mathrm{NGC}$ 1052, Cen $\mathrm{A}$ and the Milky Way}

Figure 3 shows the $\mathrm{HCO}^{+}$and $\mathrm{OH}$ optical depths which have been observed in NGC 1052 and Cen A, along with the sample of local diffuse clouds of Liszt \& Lucas (1996) and Lucas \& Liszt (2000b). As is clearly the case in Fig. 3, 


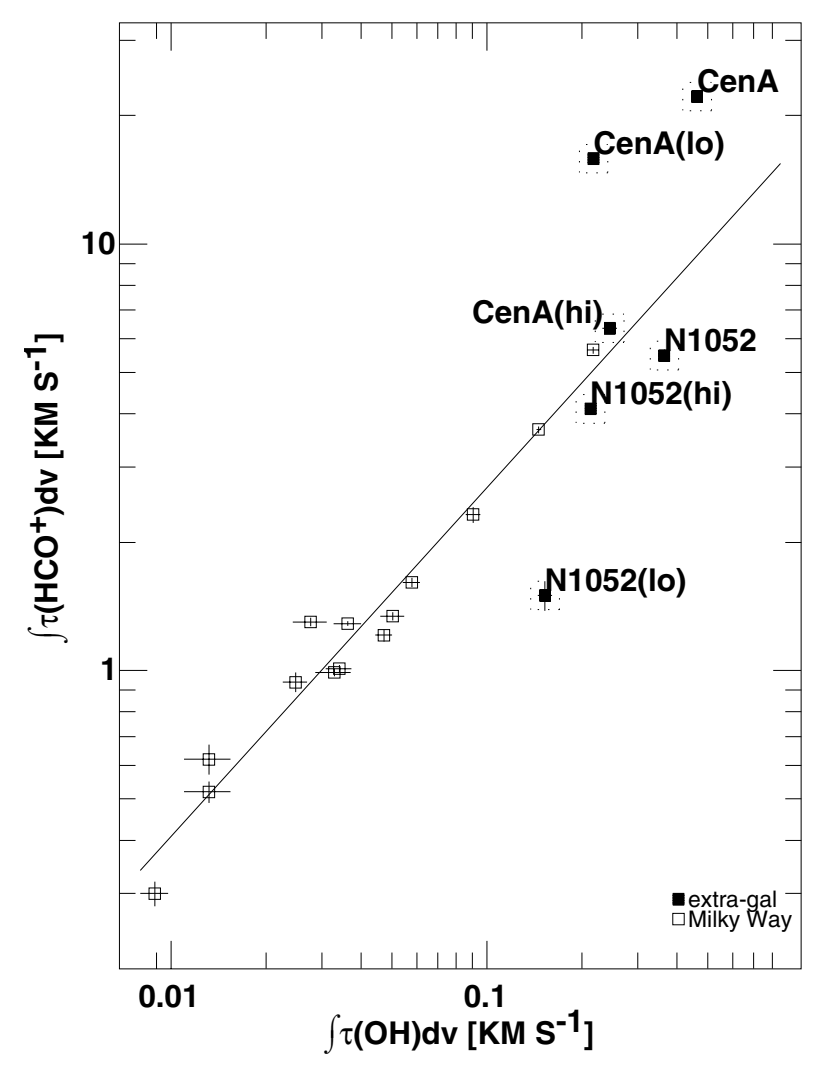

Fig. 3. $\mathrm{OH}$ and $\mathrm{HCO}^{+}$optical depths for galactic diffuse and translucent clouds, and elliptical galaxy lines of sight. The qualifications "lo" and "hi" in NGC 1052 and Cen A refer to the velocity ranges defined in Tables 1-2.

$\mathrm{OH}$ and $\mathrm{HCO}^{+}$absorption are extremely well correlated at low-to-moderate column densities. For reference, note that the $\mathrm{OH}$ column density toward $\zeta \mathrm{Oph}, N(\mathrm{OH})=5 \times 10^{13} \mathrm{~cm}^{-2}$, corresponds to an integrated opacity of approximately $0.05 \mathrm{~km} \mathrm{~s}^{-1}$. Thus the low-velocity profile integral toward NGC 1052 corresponds to little more than the amount of molecular material in a single local diffuse cloud.

The correlation between $\mathrm{OH}$ and $\mathrm{HCO}^{+}$column densities implied by our data was shown by Kanekar \& Chengalur (2002) to extend in rather spectactular fashion to four sources observed at redshifts up to about unity. By contrast, the $\mathrm{OH} / \mathrm{HCO}^{+}$integrated optical depth ratios seen in NGC 1052 and Cen A vary substantially from source to source and across the profile of either source individually. The profile integrals for lower and higher velocity gas (as defined in the footnotes to Tables 1 and 2) are shown in Fig. 3 along with the ratio of total integrals. In this plot we see that both galaxies have very similar amounts of $\mathrm{OH}$ absorption, but their $\mathrm{HCO}^{+}$integrals differ by a factor of 4 . The $\mathrm{HCO}^{+} / \mathrm{OH}$ ratios vary by similar factors of 4-5 across the profile in both systems, but the overall ratio in NGC 1052 is close to that seen in diffuse gas locally while that in Cen $\mathrm{A}$ is much larger. Not plotted, but as given in Tables $1-2$, the $\mathrm{OH} / \mathrm{H}$ I optical depth ratios actually vary little across the profile in either source, yet the $\mathrm{OH} / \mathrm{H}$ I ratio overall varies by a factor of 6 between the two systems, even more than for $\mathrm{OH} / \mathrm{HCO}^{+}$. For nearly the same amount of $\mathrm{OH}$ absorption, NGC 1052 has much smaller $\mathrm{H} \mathrm{I}$ and $\mathrm{HCO}^{+}$profile integrals.
Table 2. Integrated optical depth: Cen A (NGC 5128).

\begin{tabular}{lcccc}
\hline \hline Line & $\sigma_{l / c}$ & Low $v^{1}$ & High $v$ & Total \\
\hline & & $\mathrm{km} \mathrm{s}^{-1}$ & $\mathrm{~km} \mathrm{~s}^{-1}$ & $\mathrm{~km} \mathrm{~s}^{-1}$ \\
\hline $\mathrm{H} \mathrm{I}$ & 0.0022 & $14.03(0.02)$ & $13.53(0.02)$ & $27.43(0.03)$ \\
$\mathrm{OH}$ & 0.0020 & $0.218(0.008)$ & $0.225(0.013)$ & $0.463(0.015)$ \\
$\mathrm{HCO}^{+}$ & 0.017 & $15.83(0.18)$ & $6.34(0.10)$ & $22.16(0.21)$ \\
\hline
\end{tabular}

${ }^{1} v<565 \mathrm{~km} \mathrm{~s}^{-1}$.

For comparison we further note that the galactic $\mathrm{OH}$ absorption seen toward $\mathrm{Sgr} \mathrm{A} \mathrm{A}^{*}$ in the VLA synthesis of Killeen et al. (1992) (at $v>-70 \mathrm{~km} \mathrm{~s}^{-1}$, which omits some weak absorption arising in the $-135 \mathrm{~km} \mathrm{~s}^{-1}$ expanding molecular ring feature) has an integrated optical depth $14.6 \mathrm{~km} \mathrm{~s}^{-1}$. This is 30-40 times higher than toward either elliptical. However the Milky Way CO emission toward Sgr $\mathrm{A}^{*}$ has a profile integral of $1000 \mathrm{~K}$ in the $J=3-2$ line (Liszt \& Burton 1995), despite being heavily absorbed by intervening gas (the $J=1-0$ profile integral from the $12 \mathrm{~m}$ telescope is $1400 \mathrm{~K} \mathrm{~km} \mathrm{~s}^{-1}$ ). Thus the $\mathrm{CO}$ emission/OH absorption ratio in the Milky Way, $1000 \mathrm{~K} \mathrm{~km} \mathrm{~s}^{-1} / 14 \mathrm{~km} \mathrm{~s}^{-1} \simeq 70 \mathrm{~K}$ across the entire profile, is actually quite similar to that in Cen A, $66 \mathrm{~K} \mathrm{~km} \mathrm{~s}^{-1} / 0.463 \mathrm{~km} \mathrm{~s}^{-1}=143 \mathrm{~K}$.

\section{Summary}

The Plateau de Bure's detection of mm-wave absorption from $\mathrm{HCO}^{+}, \mathrm{HCN}$ and $\mathrm{CO}$ toward the center of the elliptical galaxy, LINER- and $\mathrm{H}_{2} \mathrm{O}$ megamaser-host NGC 1052 provides new opportunities to probe the material which obscures and reddens the optical nucleus. $\mathrm{OH}$ and $\mathrm{HCO}^{+}$yield consistent estimates of the hydrogen column density in molecular gas $2 \mathrm{~N}\left(\mathrm{H}_{2}\right) \simeq$ $5 \times 10^{21} \mathrm{~cm}^{-2}$, assuming conditions like those seen locally in the Milky Way. These estimates are quite consistent with the amount of reddening observed toward the nucleus (0.42 mag).

Our $\mathrm{HCO}^{+}$profile is somewhat broader than either $\mathrm{H} \mathrm{I}$ or $\mathrm{OH}$. Although chemical differences between $\mathrm{OH}$ and $\mathrm{HCO}^{+}$ are not expected, a wide range of other influences may be called up on account for differences in linewidths and line ratio variations across the profile. These include excitation effects, secular evolution of the background between epoochs of observation, and frequency-dependence of both the jet structure and the free-free opacity obscuring the background radio source.

$\mathrm{CO}$ emission has been unsuccessfuly sought (by others) toward NGC 1052 at fairly low levels. The absence of CO emission from gas having an optical depth of 0.1 , as observed, and a molecular column density as inferred, indicates that the molecular gas distribution is small with respect to the singledish radiotelescope beams which have been employed. Current limits on $\mathrm{CO}$ emission brightness correspond to received flux levels which are not large compared to the amount of continuum flux which is being absorbed. As for $\mathrm{H} \mathrm{I}$, attempts to observe $\mathrm{CO}$ emission toward the nucleus of NGC 1052 are seriously compromised by contamination from absorption of comparable strength.

$\mathrm{The} \mathrm{OH} / \mathrm{HCO}^{+}$ratio in NGC 1052 overall is quite close to the value established for local diffuse clouds; the gas in 
NGC 1052 is likely diffuse with a large fraction of molecular hydrogen but very incomplete conversion of carbon into $\mathrm{CO}$. The Galactic $\mathrm{OH} / \mathrm{HCO}^{+}$ratio has also been found in several sources at redshift up to about 1 . Yet, the $\mathrm{OH} / \mathrm{HCO}^{+}$ratio is markedly larger over the lower-velocity portion of the NGC 1052 absorption line profile. A comparison with observations of the other elliptical showing a similar wealth of molecular absorption lines, Cen A (NGC 5128), shows that the $\mathrm{OH} / \mathrm{HCO}^{+}$ratio also varies widely across the Cen A profile. Overall, Cen A has much more $\mathrm{H} \mathrm{I}$ and $\mathrm{HCO}^{+}$absorption than does NGC 1052, for very nearly the same OH absorption. The $\mathrm{OH}$ profile integral observed in the Milky Way toward $\mathrm{Sgr} \mathrm{A}^{*}$ is 30-40 times larger than that observed toward either NGC 1052 or NGC 5128. The reddening observed toward NGC 1052 is smaller by a similar factor (40) than that inferred toward Sgr A*.

Clarification of the distribution and kinematics of the molecular gas in the nucleus would be aided by VLB maps at frequencies comparable to (or above) those of the mm-wave absorption lines we have detected, and by searches for $\mathrm{CO}$ emission in regions of the dust lane seen away from the nuclear radiocontinuum.

Acknowledgements. The National Radio Astronomy Observatory is operated by AUI, Inc. under a cooperative agreement with the US National Science Foundation. IRAM is operated by CNRS (France), the MPG (Germany) and the IGN (Spain). We owe the staff from IRAM and at the Plateau de Bure our thanks for their assistance in taking the data. We thank Francoise Combes, Huub van Langevelde, Michael Rupen, Neil Killeen and Rene Vermeulen for providing their data in machine readable form. Comments by the referee helped us to clarify the discussion of several important issues.

\section{References}

Bland, J., Taylor, K., \& Atherton, P. D. 1987, MNRAS, 228, 595
Claussen, M. J., Diamond, P. J., Braatz, J. A., Wilson, A. S., \& Henkel, C. 1998, ApJ, 500, L129

Falcke, H., Wilson, A. S., Henkel, C., Brunthaler, A., \& Braatz, J. A. 2000, ApJ, 530, L13

Felenbok, P., \& Roueff, E. 1996, ApJ, 465, L57

Gabel, J. R., Bruhweiler, F. C., Crenshaw, D. M., Kraemer, S. B., \& Miskey, C. L. 2000, ApJ, 532, 883

Kameno, S., Sawada-Satoh, S., Inoue, M., Shen, Z., \& Wajima, K. 2001, Publ. Astron. Soc. Jpn, 53, 169

Kanekar, N., \& Chengalur, J. N. 2002, A\&A, 381, L73

Killeen, N. E. B., Lo, K. Y., \& Crutcher, R. 1992, ApJ, 385, 585

Koekemoer, A. M., Henkel, C., Greenhill, L. J., et al. 1995, Nature, 378, 697

Liszt, H. 2001, A\&A, 371, 865

Liszt, H., \& Lucas, R. 2001, A\&A, 370, 576

Liszt, H., \& Lucas, R. 2002, A\&A, 391, 693

Liszt, H. S., \& Burton, W. B. 1995, ApJS, 98, 679

Liszt, H. S., \& Lucas, R. 1996, A\&A, 314, 917

Liszt, H. S., \& Lucas, R. 1998, A\&A, 339, 561

Liszt, H. S., \& Lucas, R. 2000, A\&A, 355, 333

Lucas, R., \& Liszt, H. S. 1996, A\&A, 307, 237

Lucas, R., \& Liszt, H. S. 2000a, A\&A, 358, 1069

Lucas, R., \& Liszt, H. S. 2000b, A\&A, 355, 327

Omar, A., Anantharamaiah, K. R., Rupen, M., \& Rigby, J. 2002, A\&A, 381, L29

Pei, Y. C., Fall, S. M., \& Bechtold, J. 1991, ApJ, 378, 6

Plana, H., \& Boulesteix, J. 1996, A\&A, 307, 391

Sarma, A. P., Troland, T. H., \& Rupen, M. P. 2002, ApJ, 564, 696

Savage, B. D., \& Sembach, K. R. 1996, ARA\&A, 34, 279

van Gorkom, J. H., Knapp, G. R., Raimond, E., Faber, S. M., \& Gallagher, J. S. 1986, AJ, 91, 791

van Langevelde, H. J., van Dishoeck, E. F., Sevenster, M. N., \& Israel, F. P. 1995, ApJ, 448, L123

Vermeulen, R. C., Ros, E., Kellermann, K. I., et al. 2003, A\&A, 401, 113

Wiklind, T., \& Combes, F. 1997, A\&A, 324, 51

Wiklind, T., Combes, F., \& Henkel, C. 1995, A\&A, 297, 643

Wrobel, J. M. 1984, ApJ, 284, 531 